\title{
Parental perception of child weight and its association with weight-related parenting behaviours and child behaviours: a Chinese national study
}

\author{
Ting Zhang ${ }^{1} \dagger$, Li Cai ${ }^{1}{ }^{\dagger}$, Jin Jing ${ }^{1}$, Lu Ma ${ }^{1}$, Jun $\mathrm{Ma}^{2, *}$ and Yajun Chen ${ }^{1, *}$ \\ 'Department of Maternal and Child Health, School of Public Health, Sun Yat-Sen University, Guangzhou 510080, \\ People's Republic of China: ${ }^{2}$ Institute of Child and Adolescent Health, School of Public Health, Peking University, \\ Beijing 100191, People's Republic of China
}

Submitted 5 June 2017: Final revision received 23 November 2017: Accepted 2 January 2018: First published online 7 March 2018

\begin{abstract}
Objective: Parents commonly fail to correctly recognize the weight status of their child. Whether parental perception of child weight is associated with weightrelated parenting behaviours and child behaviours is unclear. The present study aimed to investigate the distribution of parental perception of child weight and its associations with weight-related parenting behaviours and child behaviours in China.

Design: Cross-sectional study.

Setting: Seven provinces in China.

Subjects: A total of 47417 children aged 6-17 years and their parents were included from a national survey in 2013. Parental perception of child weight, weight-related parenting behaviours and child behaviours were self-reported. Child's weight and height were objectively measured.

Results: A total of $30.5 \%$ of parents underestimated and $8.7 \%$ overestimated the child's weight. Parental underestimation was more common among younger children, boys and children with a lower BMI $Z$-score. Parents who perceived that their child had a healthy weight (accurately or inaccurately) were more likely to prepare breakfast for the child, exercise with him/her, set apart his/her exercise time, restrict his/her screen time, and were less likely to store soft drinks for the child. Children perceived to have a healthy weight, regardless of their actual weight status, behaved healthier on dietary intake, physical activity and homework time.

Conclusions: Parental underestimation of their child's weight was prominent in China, especially among younger children, boys and children with a lower BMI $Z$-score. Parental recognition of their child being overweight did not appear to translate into healthy changes in weight-related parenting behaviours or child behaviours.
\end{abstract}

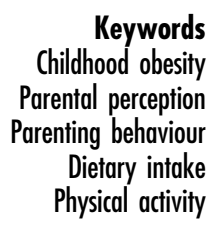

Childhood obesity remains a global public health issue ${ }^{(1)}$. A target of obesity interventions is the promotion of healthy lifestyle changes, including diet, physical activity and sedentary behaviour of children. Given that parents are influential in modelling eating behaviours ${ }^{(2)}$ and physical activity for their children, parents' active participation is important in effective childhood obesity interventions. The perception that their child is overweight or obese should be an important step for parents to act in their child's weight-management programme ${ }^{(3)}$. However, previous studies have affirmed that large proportions of

$\dagger$ Ting Zhang and Li Cai contributed equally to this work. parents fail to recognize that their child is overweight or obese $^{(4-7)}$. For instance, a meta-analysis showed that half of parents underestimated the overweight/obese status of their child ${ }^{(7)}$. The correlates of parental misperception of child weight include child's age ${ }^{(6-8)}$, gender ${ }^{(7-10)}$, ethnicity $^{(11)}$, BMI ${ }^{(7,10,12)}$, parental education level ${ }^{(12)}$, parental weight status ${ }^{(7-9)}$ and socio-economic status ${ }^{(11)}$, which indicates that the extent of this misperception varies in different populations.

Given that parental underestimation of their child's weight is prevalent, it is important to determine whether this underestimation affects weight-related parenting behaviours and child behaviours, thus influencing the 
child's weight status. However, a few studies have found controversial results on the association of parental perception with parenting behaviours or child behaviours $^{(12-14)}$. A study of 482 overweight pre-school children and their mothers in Kuwait found that the mothers' accurate perception of their child's weight was associated with their plans to control the weight of their pre-school child ${ }^{(13)}$. Another study in the UK targeting 361 children aged 6-8 years and their parents reported that the children whose weight status was misperceived by their parents tended to get a lower score on a 'healthy' dietary pattern than those who were correctly perceived ${ }^{(14)}$. However, a study consisting of 783 overweight and obese 5-year-old children and their parents in the Netherlands demonstrated that parental misperception of the weight of their child was not associated with their intention to improve weight-related behaviours of their child or with the child meeting the guidelines for these behaviours ${ }^{(12)}$. Considering the crucial role of parents in family-based obesity interventions ${ }^{(15)}$, the unclear relationship between parental perception and weight-related parenting behaviours or child behaviours should be explored.

Only a few studies with a small sample size ${ }^{(16-18)}$ have reported Chinese parents' perception of their child's weight, which may be distinct from those in Western countries due to different ethnicities, socio-economic status and cultural contexts. A survey of children aged 6-18 years showed that $72 \%$ of mothers underestimated the overweight status of their child ${ }^{(17)}$. Furthermore, only one study examining the relationship between parental perceptions and parenting behaviours in adolescents suggested that parents with correct perception were more likely to monitor the diet and physical activity of their child and gave more positive reinforcement for manifesting healthy behaviours than those who were wrong perceivers $^{(18)}$. The related situation in Chinese primary-school children is still unknown, the parents of whom might play even more important roles in modelling the child's behaviours in the family. Using a national large sample, our study aimed to investigate parental perception of their child's weight and its demographic correlates, and to examine the associations between parental perception and weight-related parenting behaviours and child behaviours among Chinese children.

\section{Methods}

\section{Study design and participants}

The present study was a baseline survey of a multicentre school-based obesity intervention programme among Chinese children (trial registration number: NCT02343588) ${ }^{(19,20)}$ conducted between September and November 2013. The protocol of the study was reviewed and approved by the Ethics Committee of Peking University. All participating children and their parents signed informed consent forms.
Multistage random cluster sampling was applied in the recruitment. First, seven provinces in China (i.e. Liaoning, Tianjin, Ningxia, Shanghai, Chongqing, Hunan and Guangdong) were selected using the judgement sampling method. Second, schools were stratified by district and school size and then twelve to seventeen primary and secondary schools were randomly selected through the probability-proportional-to-size sampling method in each of the selected provinces. With the agreement of the principals, all students in grades $1-5,7-8$ and $10-11$ were invited to participate (students in grades 6, 9 and 12 were not contacted due to study load). In total, 65347 students from fifty primary schools and forty-five secondary schools and their parents participated in the study. We excluded students with age $<6$ years ( $n$ 193) or $\geq 18$ years $(n 346)$, those who lacked data about BMI ( $n$ 2291) or parental perception of child weight ( $n$ 10696), and those whose parent questionnaire was not filled out by the parents ( $n$ 4404). Finally, 47417 students and their parents were included in the sample for analyses. No significant difference was observed in child age or gender between those in the analytic sample and those who were excluded.

\section{Measurements}

Standardized questionnaires were used to measure sociodemographic characteristics, perception of weight status from parents and child, weight-related parenting behaviours and child weight-related behaviours. All questionnaires were delivered in class by trained investigators and were filled out by both parents and children. The questionnaires had been piloted and revised before the study, being found to be sufficiently reliable and acceptable to the children and their parents ${ }^{(20)}$.

\section{Sociodemographic characteristics}

Child birth date, gender, parental education level, current height (in centimetres), current weight (in kilograms) and monthly family income were evaluated in the questionnaires. BMI was calculated as weight (in kilograms) divided by the square of height (in metres). Parental underweight, overweight and obesity were defined using the Chinese criteria for adults ${ }^{(21)}$ as BMI $<18.5 \mathrm{~kg} / \mathrm{m}^{2}$, BMI $\geq 24$ but $<28 \mathrm{~kg} / \mathrm{m}^{2}$ and $\mathrm{BMI} \geq 28 \mathrm{~kg} / \mathrm{m}^{2}$, respectively.

\section{Perception of weight status from parent and child}

Parental perception of child weight was assessed by asking: 'How do you feel about current weight status of your child?' ('thin', 'about the right weight' or 'fat'). The responses were explained as perceived underweight ('thin'), healthy weight ('about the right weight') and overweight ('fat'). Child self-perception of weight status was assessed by asking: 'How do you feel about your current weight status?' ('very thin', 'rather thin', 'average', 'rather fat' or 'very fat'). The responses were explained as perceived underweight ('very thin' or 'rather thin'), 
healthy weight ('average') and overweight ('very fat' or 'rather fat'). Parental and child perceptions were compared with the child's actual weight status to define underestimation, accurate estimation and overestimation of child weight.

\section{Weight-related parenting behaviours}

Parents responded to the following questions: (i) 'Did you prepare breakfast for your child in the past week?'; (ii) 'Did you store soft drinks (e.g. cola and juice) at home in the past week?'; (iii) 'Did you buy soft drinks for your child in the past week?'; (iv) 'Did you restrict exercise time of your child such that he/she had more time for homework or other important things in the past week?'; (v) 'Did you set apart exercise time for your child in the past week?'; and (vi) 'Did you restrict screen time of your child in the past week?' The response options were 'never', 'seldom', 'sometimes', 'often' and 'always' for questions (i) to (v), and 'yes' or 'no' for question (vi). Parents also reported daily time of exercise with their child over the past week.

\section{Child's weight-related behaviours}

Dietary intake, screen time, homework time and physical activity in the past seven consecutive days were evaluated using the questionnaires. The children reported the frequency (days) and amount (servings) of fruits, vegetables, meat products and soft drinks; and frequency (days or times) of breakfast, high-energy snack (e.g. chocolates and candies), fried food (e.g. fried chicken and fried pancake) and Western fast food (e.g. KFC and McDonald's) consumption. The mean daily intakes of fruits, vegetables, meat products and soft drinks were calculated by the formula: mean daily intake $=[$ days $\times$ (amount in each of those days)]/7. Screen time was assessed by summarizing the daily time of viewing television and using computers. Homework time was assessed by asking daily time of doing work after school. Moderate-to-vigorous physical activity (MVPA) was measured by summarizing the daily time of vigorous (e.g. running and basketball) and moderate (e.g. cycling and badminton) physical activities.

\section{Child's actual weight status}

The children's height (in centimetres) and weight (in kilograms) were measured by trained technicians in a standardized procedure. Height was measured using a portable stadiometer (Yilian TZG, China), whereas weight was measured using a lever scale (Hengxing RGT-140, China). BMI was calculated and the age- and genderspecific cut-offs of BMI for malnutrition ${ }^{(22)}$ and overweight (including obesity) $^{(23)}$ of Chinese children were used for the classification of weight status. Given that the Chinese criteria for overweight ${ }^{(23)}$ do not include cut-offs for children aged 6 years, we used the WHO standards ${ }^{(24)}$ to define overweight for this population. BMI $Z$-score of the children was calculated using the WHO standards ${ }^{(25)}$.

\section{Statistical analysis}

All statistical analyses were performed using the statistical software package IBM SPSS Statistics version 19.0. The continuous and categorical variables were presented as mean and standard deviation, and as number and percentage, respectively. Differences in demographic variables between boys and girls were evaluated via Pearson $\chi^{2}$ tests. Correlates of parental underestimation of child weight ( $v$. accurate perception) were assessed through binary logistic regression analysis, adjusting for child's age, gender, BMI $Z$-score, area, parental education level, monthly family income, parental weight status, region and school. For children of healthy weight, those who were overestimated by parents were excluded in the regression analyses. Binary logistic regression analyses were performed to evaluate the associations of parental perception of child weight with weight-related parenting behaviours and child behaviours, adjusting for child's age, gender, region and school. A two-tailed $P<0.05$ was considered statistically significant in all analyses.

\section{Results}

The present study included 47417 children aged 6-17 years ( $49.4 \%$ girls) and their parents. Table 1 presents the characteristics of the participants. The prevalence of underweight and overweight (including obesity) was 6.6 and $24.7 \%$, respectively. Overall, $30.5 \%$ of the parents underestimated and $8.7 \%$ overestimated the weight status of their child. Parental underestimation was prevalent among healthy-weight (29.1\%) and overweight children ( $42.9 \%)$. The weight of the boys was more likely to be underestimated than that of the girls $(35.8 v$. 25.1\%, $P<0.001$ ). Only $68.4 \%$ of the children had concordant perceptions with their parents, and $46 \cdot 2 \%$ of both children and parents had accurate assessment of the child's weight. The parents provided slightly more accurate assessment than the children themselves $(60.8 v .59 \cdot 8 \%, P<0.001$; Table 2). The proportion of parental underestimation decreased and the proportion of accurate estimation increased for children in the higher age group $\left(P_{\text {trend }}<\right.$ 0.001; see online supplementary material, Supplemental Fig. 1). When using the WHO standards for underweight and overweight ${ }^{(24)}$ in all children, the prevalence of underweight and overweight (including obesity) was 2.9 and $26.9 \%$, respectively, and $34.8 \%$ of the parents underestimated and $7 \cdot 4 \%$ overestimated the weight of their child (Supplemental Table 1).

For both healthy-weight and overweight children respectively, older age (adjusted OR (AOR) $=0.90,95 \% \mathrm{CI}$ $0.88,0.92$ and $\mathrm{AOR}=0.76,95 \%$ CI 0.74, 0.79), girls (v. boys, $\mathrm{AOR}=0.40,95 \%$ CI 0.38, 0.42 and $\mathrm{AOR}=0.38$, $95 \%$ CI $0.34,0 \cdot 41)$ and higher BMI $Z$-score $(\mathrm{AOR}=0 \cdot 19$, $95 \%$ CI $0 \cdot 18,0 \cdot 20$ and $\mathrm{AOR}=0 \cdot 18,95 \%$ CI 0.16, 0.20) were negatively associated with parental underestimation of 
Table 1 Sociodemographic characteristics of the study population of children aged $6-17$ years and their parents $(n 47417)$ from seven provinces in China, September-November 2013

\begin{tabular}{|c|c|c|c|c|c|c|c|}
\hline \multirow[b]{2}{*}{ Characteristic } & \multicolumn{2}{|c|}{ Total } & \multicolumn{2}{|c|}{ Boys $(50.7 \%)$} & \multicolumn{2}{|c|}{ Girls $(49 \cdot 3 \%)$} & \multirow[b]{2}{*}{$P \dagger$} \\
\hline & $n$ & $\%$ & $n$ & $\%$ & $n$ & $\%$ & \\
\hline Age (years) & & & & & & & $<0.001$ \\
\hline $6.0-8.9$ & 15451 & 32.6 & 8037 & 33.5 & 7414 & 31.6 & \\
\hline $9.0-11.9$ & 12096 & 25.5 & 6441 & $26 \cdot 9$ & 5655 & $24 \cdot 1$ & \\
\hline $12 \cdot 0-14.9$ & 11423 & $24 \cdot 1$ & 5665 & 23.6 & 5758 & $24 \cdot 6$ & \\
\hline $15 \cdot 0-17.9$ & 8447 & $17 \cdot 8$ & 3828 & $16 \cdot 0$ & 4619 & $19 \cdot 7$ & \\
\hline Area & & & & & & & 0.688 \\
\hline Urban & 29225 & $61 \cdot 6$ & 14753 & 61.5 & 14472 & $61 \cdot 7$ & \\
\hline Rural & 18192 & 38.4 & 9218 & 38.5 & 8974 & 38.3 & \\
\hline Paternal education level & & & & & & & 0.092 \\
\hline Junior high school or below & 19556 & $42 \cdot 2$ & 9992 & $42 \cdot 6$ & 9564 & 41.7 & \\
\hline Senior high school or junior college & 19916 & $42 \cdot 9$ & 10023 & $42 \cdot 7$ & 9893 & $43 \cdot 1$ & \\
\hline College or above & 6909 & $14 \cdot 9$ & 3433 & $14 \cdot 6$ & 3476 & $15 \cdot 2$ & \\
\hline Maternal education level & & & & & & & 0.033 \\
\hline Junior high school or below & 21319 & $46 \cdot 0$ & 10910 & $46 \cdot 6$ & 10409 & 45.4 & \\
\hline Senior high school or junior college & 19368 & 41.8 & 9676 & 41.3 & 9692 & $42 \cdot 3$ & \\
\hline College or above & 5656 & $12 \cdot 2$ & 2826 & $12 \cdot 1$ & 2830 & $12 \cdot 3$ & \\
\hline Monthly family income & & & & & & & 0.030 \\
\hline$<5000 \mathrm{RMB}$ & 12824 & $31 \cdot 2$ & 6238 & $30 \cdot 6$ & 6586 & 31.9 & \\
\hline 5000-8000 RMB & 7790 & $19 \cdot 0$ & 3916 & $19 \cdot 2$ & 3874 & 18.8 & \\
\hline$\geq 8000 \mathrm{RMB}$ & 8607 & $21 \cdot 0$ & 4313 & $21 \cdot 1$ & 4294 & $20 \cdot 8$ & \\
\hline Refuse to disclose & 11816 & 28.8 & 5942 & $29 \cdot 1$ & 5874 & 28.5 & \\
\hline Paternal weight status & & & & & & & 0.139 \\
\hline Underweight & 997 & $2 \cdot 2$ & 479 & $2 \cdot 1$ & 518 & $2 \cdot 3$ & \\
\hline Healthy weight & 21734 & 48.4 & 10941 & $48 \cdot 1$ & 10793 & $48 \cdot 7$ & \\
\hline Overweight & 17058 & 38.0 & 8667 & $38 \cdot 1$ & 8391 & $37 \cdot 8$ & \\
\hline Obese & 5121 & 11.4 & 2642 & 11.6 & 2479 & 11.2 & \\
\hline Maternal weight status & & & & & & & 0.003 \\
\hline Underweight & 3223 & $7 \cdot 2$ & 1707 & 7.5 & 1516 & $6 \cdot 8$ & \\
\hline Healthy weight & 31528 & $70 . \overline{0}$ & 15951 & $70 \cdot 2$ & 15577 & 69.9 & \\
\hline Overweight & 8568 & $19 \cdot 0$ & 4261 & $18 \cdot 7$ & 4307 & $19 \cdot 3$ & \\
\hline Obese & 1692 & 3.8 & 814 & 3.6 & 878 & 3.9 & \\
\hline
\end{tabular}

†Differences in characteristic variables between boys and girls were evaluated using Pearson $x^{2}$ tests; $P<0.05$ indicates significance.

Table 2 Parental perception of their child's weight by the child's self-perception and measured weight status; children aged 6-17 years and their parents $(n$ 47 417) from seven provinces in China, September-November 2013

\begin{tabular}{|c|c|c|c|c|c|c|c|c|}
\hline \multirow[b]{3}{*}{ Parental perception } & \multicolumn{6}{|c|}{ Child's self-perception of weight } & & \\
\hline & \multicolumn{2}{|c|}{ Underweight } & \multicolumn{2}{|c|}{ Healthy weight } & \multicolumn{2}{|c|}{ Overweight } & \multicolumn{2}{|c|}{ Total } \\
\hline & $n$ & $\%$ & $n$ & $\%$ & $n$ & $\%$ & $n$ & $\%$ \\
\hline \multicolumn{9}{|c|}{ Measured underweight ( $n$ 3144) } \\
\hline Underweight & 2048 & 67.9 & 186 & $6 \cdot 2$ & 18 & 0.6 & 2326 & 74.0 \\
\hline Healthy weight & 369 & $12 \cdot 2$ & 188 & $6 \cdot 2$ & 12 & 0.4 & 606 & 19.3 \\
\hline Overweight & 158 & $5 \cdot 2$ & 22 & 0.7 & 17 & 0.6 & 212 & 6.7 \\
\hline \multicolumn{9}{|c|}{ Measured healthy weight ( $n$ 32 564) } \\
\hline Underweight & 6470 & $20 \cdot 7$ & 2280 & $7 \cdot 3$ & 311 & 1.0 & 9462 & 29.1 \\
\hline Healthy weight & 3383 & $10 \cdot 8$ & 13203 & $42 \cdot 3$ & 2510 & 8.0 & 19795 & $60 \cdot 8$ \\
\hline Overweight & 727 & $2 \cdot 3$ & 1206 & 3.9 & 1150 & 3.7 & 3307 & $10 \cdot 2$ \\
\hline \multicolumn{9}{|c|}{ Measured overweight ( $n$ 11709) } \\
\hline Underweight & 71 & 0.6 & 147 & 1.3 & 303 & $2 \cdot 7$ & 561 & 4.8 \\
\hline Healthy weight & 142 & 1.3 & 2208 & 19.7 & 1911 & $17 \cdot 0$ & 4461 & 38.1 \\
\hline Overweight & 73 & 0.7 & 631 & 5.6 & 5743 & 51.1 & 6687 & $57 \cdot 1$ \\
\hline \multicolumn{9}{|l|}{ Overall ( $n$ 47 417) } \\
\hline Underweight & 8589 & 18.9 & 2613 & $5 \cdot 7$ & 632 & 1.4 & 12349 & $26 \cdot 0$ \\
\hline Healthy weight & 3894 & 8.6 & 15599 & $34 \cdot 3$ & 4433 & $9 \cdot 7$ & 24862 & $52 \cdot 4$ \\
\hline Overweight & 958 & $2 \cdot 1$ & 1859 & $4 \cdot 1$ & 6910 & $15 \cdot 2$ & 10206 & 21.5 \\
\hline
\end{tabular}

their child's weight. Furthermore, healthy-weight children were more likely to be underestimated if their respective mothers were underweight $(\mathrm{AOR}=1 \cdot 15,95 \%$ CI $1 \cdot 03$, 1.27; Table 3).
Table 4 shows the associations between parental perception of child weight and weight-related parenting behaviours. In total, a large majority of the parents reported that they prepared breakfast for their child 
Table 3 Correlates of parental underestimation of their child's weight ( $v$. accurate estimation); children aged 6-17 years and their parents ( $n$ 47417) from seven provinces in China, September-November 2013

\begin{tabular}{|c|c|c|c|c|c|c|c|c|}
\hline & \multicolumn{4}{|c|}{ Healthy-weight children ( $n$ 29257)† } & \multicolumn{4}{|c|}{ Overweight children ( $n$ 11709) } \\
\hline & \multicolumn{2}{|c|}{ Crude model } & \multicolumn{2}{|c|}{ Adjusted model $\ddagger$} & \multicolumn{2}{|c|}{ Crude model } & \multicolumn{2}{|c|}{ Adjusted model $\ddagger$} \\
\hline & OR & $95 \% \mathrm{Cl}$ & OR & $95 \% \mathrm{Cl}$ & OR & $95 \% \mathrm{Cl}$ & OR & $95 \% \mathrm{Cl}$ \\
\hline Age (years) & $0.92^{\star *}$ & $0.91,0.92$ & $0.90^{\star \star}$ & $0.88,0.92$ & $0.93^{\star \star}$ & $0.91,0.94$ & 0.76 & $0.74,0.79$ \\
\hline \multicolumn{9}{|l|}{ Gender } \\
\hline Boys & 1.00 & Ref. & 1.00 & Ref. & 1.00 & Ref. & 1.00 & Ref. \\
\hline Girls & $0.58^{\star \star}$ & $0.55,0.60$ & $0.40^{* *}$ & $0.38,0.42$ & $0.80^{\star \star}$ & $0.74,0.86$ & $0.38^{\star *}$ & $0.34,0.41$ \\
\hline Child BMI Z-score & $0.25^{\star \star}$ & $0.24,0.26$ & $0.19^{\star \star}$ & $0.18,0.20$ & $0.35^{\star \star}$ & $0.32,0.37$ & $0.18^{\star *}$ & $0.16,0.20$ \\
\hline \multicolumn{9}{|l|}{ Area } \\
\hline Urban & 1.03 & $0.98,1.09$ & 0.81 & $0.58,1.13$ & 1.05 & $0.98,1.13$ & 1.02 & $0.47,2.20$ \\
\hline Rural & 1.00 & Ref. & 1.00 & Ref. & 1.00 & Ref. & 1.00 & Ref. \\
\hline \multicolumn{9}{|l|}{ Paternal education level } \\
\hline Junior high or below & 1.00 & Ref. & 1.00 & Ref. & 1.00 & Ref. & 1.00 & Ref. \\
\hline Senior high or junior college & $1.08^{\star \star}$ & $1.02,1.13$ & 1.07 & $0.99,1.16$ & 0.95 & $0.87,1.03$ & 1.01 & $0.89,1.14$ \\
\hline College or above & $1.08^{*}$ & $1.01,1.17$ & $1 \cdot 10$ & $0.98,1.25$ & 1.05 & $0.94,1.17$ & 1.02 & $0.85,1.22$ \\
\hline \multicolumn{9}{|l|}{ Maternal education level } \\
\hline Junior high or below & 1.00 & Ref. & 1.00 & Ref. & 1.00 & Ref. & 1.00 & Ref. \\
\hline Senior high or junior college & 1.03 & $0.98,1.09$ & 0.96 & $0.89,1.04$ & 1.02 & $0.95,1.11$ & 1.06 & $0.94,1.20$ \\
\hline College or above & 1.01 & $0.93,1.09$ & 0.92 & $0.81,1.04$ & $1 \cdot 17^{*}$ & $1.04,1.31$ & 1.11 & $0.92,1.35$ \\
\hline \multicolumn{9}{|l|}{ Monthly family income } \\
\hline$<5000 \mathrm{RMB}$ & 1.00 & Ref. & 1.00 & Ref. & 1.00 & Ref. & 1.00 & Ref. \\
\hline 5000-8000 RMB & 1.06 & $0.99,1.15$ & 0.97 & $0.89,1.07$ & $0.80^{\star *}$ & $0.72,0.90$ & 0.92 & $0.80,1.06$ \\
\hline$\geq 8000 \mathrm{RMB}$ & $1 \cdot 13^{\star \star}$ & $1.05,1.22$ & 0.99 & $0.90,1.09$ & $0.71^{\star *}$ & $0.64,0.79$ & 0.96 & $0.83,1.12$ \\
\hline Refuse to answer & 1.05 & $0.98,1.13$ & 0.98 & $0.90,1.06$ & $0.79^{\star \star}$ & $0.72,0.88$ & 0.99 & $0.87,1.12$ \\
\hline \multicolumn{9}{|l|}{ Paternal weight status } \\
\hline Underweight & $1 \cdot 15$ & $0.98,1.36$ & 1.05 & $0.87,1.26$ & 0.92 & $0.66,1.27$ & 1.02 & $0.69,1.52$ \\
\hline Healthy weight & 1.00 & Ref. & 1.00 & Ref. & 1.00 & Ref. & 1.00 & Ref. \\
\hline Overweight & $0.86^{\star \star}$ & $0.81,0.91$ & 1.05 & $0.98,1.12$ & $0.85^{\star *}$ & $0.78,0.92$ & 0.91 & $0.82,1.01$ \\
\hline Obese & $0.66^{\star *}$ & $0.60,0.73$ & 0.90 & $0.81,1.01$ & $0.77^{\star \star}$ & $0.69,0.86$ & 0.88 & $0.77,1.00$ \\
\hline \multicolumn{9}{|l|}{ Maternal weight status } \\
\hline Underweight & $1.53^{\star *}$ & $1.40,1.68$ & $1 \cdot 15^{\star}$ & $1.03,1.27$ & $1 \cdot 14$ & $0.94,1.37$ & 0.95 & $0.76,1.20$ \\
\hline Healthy weight & 1.00 & Ref. & 1.00 & Ref. & 1.00 & Ref. & 1.00 & Ref. \\
\hline Overweight & $0.75^{\star \star}$ & $0.70,0.81$ & 1.02 & $0.94,1.10$ & $0.90^{*}$ & $0.83,0.99$ & 1.03 & $0.93,1.15$ \\
\hline Obese & $0.74^{\star \star}$ & $0.63,0.87$ & $1 \cdot 10$ & $0.92,1.32$ & $0.74^{\star *}$ & $0.63,0.87$ & 0.99 & $0.81,1.21$ \\
\hline
\end{tabular}

Ref., referent category.

OR and $95 \% \mathrm{Cl}$ were derived from binary logistic regression analysis.

${ }^{*} P<0.05,{ }^{* *} P<0.01$.

†Healthy-weight children who were overestimated by parents are excluded from the analysis ( $n 3684,10.3 \%$ of healthy-weight children).

$\ddagger$ Adjusted for each other, region and school.

(90.0\%) and restricted their screen time (92.4\%). Over half of the parents bought soft drinks for their child (55.9\%) and nearly one-third stored soft drinks at home $(31 \cdot 1 \%)$. Of the parents, $56.3 \%$ never or seldom restricted exercise time of their child. A substantial proportion of the parents set apart exercise time for their child $(81 \cdot 1 \%)$ and $57 \cdot 1 \%$ of parents exercised with their child over the past week. Parental perception that their child was of healthy weight, regardless of the actual weight status, was positively associated with exercising with the child and setting apart the child's exercise time. For underweight children, parental overestimation was negatively associated with buying soft drinks for the child. For healthy-weight children, accurate parental perception was positively associated with parents preparing breakfast for the child and restricting their screen time and negatively associated with storing soft drinks at home. For overweight children, parental underestimation was positively associated with parents preparing breakfast for them.
The majority of the children consumed $\leq 1$ serving meat products/d (73.1\%), had breakfast every day (84.8\%), and spent $\leq 2 \mathrm{~h} / \mathrm{d}$ on screen time $(77.2 \%)$ and $\leq 2 \mathrm{~h} / \mathrm{d}$ on homework time $(66 \cdot 8 \%)$. But only a minority of children consumed $\geq 2$ servings fruits/d (25.6\%), $\geq 3$ servings vegetables/d (19.6\%) or accomplished $\geq 1 \mathrm{~h}$ MVPA/d $(31 \cdot 2 \%)$. A substantial proportion of children consumed soft drinks (33.9\%), high-energy snacks (25.5\%), fried food (43.0\%) and Western fast foods (51.9\%). Parental accurate estimation of healthy-weight children was positively associated with the child consuming $\geq 2$ servings fruits/d and $\leq 1$ serving meat products/d, having breakfast every day, avoiding fried food, accomplishing $\geq 1 \mathrm{~h}$ MVPA/d and spending $\leq 2 \mathrm{~h} / \mathrm{d}$ on screen time and $\leq 2 \mathrm{~h} / \mathrm{d}$ on homework time. In addition, parental underestimation of overweight children was positively associated with the child consuming $\leq 1$ serving meat products/d, avoiding soft drinks and spending $\leq 2 \mathrm{~h} / \mathrm{d}$ on screen time, but negatively associated with avoiding Western fast foods (Table 5). 
Table 4 Associations between parental perception of their child's weight and weight-related parenting behaviours; children aged 6-17 years and their parents ( $n$ 47417) from seven provinces in China, September-November 2013

\begin{tabular}{|c|c|c|c|c|c|c|c|c|c|c|c|c|}
\hline \multirow[b]{3}{*}{ Behaviour items } & & & \multicolumn{6}{|c|}{ Parental perception } & & & & \\
\hline & \multicolumn{2}{|c|}{ Total } & \multicolumn{2}{|c|}{ Underestimate } & \multicolumn{2}{|c|}{ Accurate } & \multicolumn{2}{|c|}{ Overestimate } & \multicolumn{2}{|c|}{ Underestimate } & \multicolumn{2}{|c|}{ Overestimate } \\
\hline & $n$ & $\%$ & $n$ & $\%$ & $n$ & $\%$ & $n$ & $\%$ & OR & $95 \% \mathrm{Cl}$ & OR & $95 \% \mathrm{Cl}$ \\
\hline \multicolumn{13}{|l|}{ Underweight children ( $n$ 3144) } \\
\hline $\begin{array}{l}\text { Prepares breakfast for child } \\
\text { (always, often or sometimes) }\end{array}$ & 2861 & $91 \cdot 6$ & \multicolumn{2}{|c|}{-} & 2116 & 91.5 & 745 & $91 \cdot 6$ & & - & 1.08 & $0.77,1.53$ \\
\hline $\begin{array}{l}\text { Stores soft drink at home } \\
\text { (never or seldom) }\end{array}$ & 2167 & $69 \cdot 2$ & \multicolumn{2}{|c|}{-} & 1610 & $69 \cdot 5$ & 557 & $68 \cdot 4$ & & - & $1 \cdot 10$ & $0.90,1.36$ \\
\hline $\begin{array}{l}\text { Buys soft drinks for child } \\
\text { (never or seldom) }\end{array}$ & 1342 & $42 \cdot 9$ & \multicolumn{2}{|c|}{-} & 983 & $42 \cdot 5$ & 359 & $44 \cdot 2$ & & - & $1 \cdot 25^{\star}$ & $1.03,1.52$ \\
\hline Exercises with child $(>0 \mathrm{~h} / \mathrm{d})$ & 1671 & $56 \cdot 3^{\star}$ & \multicolumn{2}{|c|}{-} & 1219 & $55 \cdot 1$ & 452 & 59.9 & & - & $1.43^{\star *}$ & $1 \cdot 16,1 \cdot 77$ \\
\hline $\begin{array}{l}\text { Restricts child's exercise time } \\
\text { (never or seldom) }\end{array}$ & 1708 & $54 \cdot 7$ & \multicolumn{2}{|c|}{-} & 1270 & 54.9 & 438 & $53 \cdot 9$ & & - & 1.07 & $0.88,1.30$ \\
\hline $\begin{array}{l}\text { Sets apart child's exercise time } \\
\text { (always, often or sometimes) }\end{array}$ & 2510 & $80 \cdot 6^{* *}$ & \multicolumn{2}{|c|}{-} & 1827 & $79 \cdot 1$ & 683 & $84 \cdot 8$ & & - & $1.49^{\star *}$ & $1.15,1.94$ \\
\hline \multicolumn{11}{|c|}{ Healthy-weight children ( $n$ 32564) } & & \\
\hline $\begin{array}{l}\text { Prepares breakfast for child } \\
\text { (always, often or sometimes) }\end{array}$ & 29119 & $90 \cdot 0^{* *}$ & 8467 & $90 \cdot 0$ & 17772 & $90 \cdot 4$ & 2880 & $87 \cdot 5$ & 0.95 & $0.87,1.03$ & $0.79^{* *}$ & $0.70,0.89$ \\
\hline $\begin{array}{l}\text { Stores soft drinks at home } \\
\text { (never or seldom) }\end{array}$ & 22355 & $69 \cdot 0^{*}$ & 6476 & $68 \cdot 8$ & 13663 & 69.4 & 2216 & $67 \cdot 3$ & 0.95 & $0.90,1.00$ & $0.90^{* *}$ & $0.83,0.97$ \\
\hline $\begin{array}{l}\text { Buys soft drinks for child } \\
\text { (never or seldom) }\end{array}$ & 14174 & $43 \cdot 7$ & 4128 & $43 \cdot 8$ & 8623 & $43 \cdot 8$ & 1423 & $43 \cdot 2$ & 0.96 & $0.91,1.01$ & 1.02 & $0.94,1.10$ \\
\hline Exercises with child $(>0 \mathrm{~h} / \mathrm{d})$ & 17298 & $56 \cdot 1^{\star *}$ & 5002 & $55 \cdot 8$ & 10697 & $57 \cdot 2$ & 1599 & $50 \cdot 6$ & $0 \cdot 80^{\star *}$ & $0.75,0.84$ & $0.90^{*}$ & $0.83,0.98$ \\
\hline $\begin{array}{l}\text { Restricts child's exercise time } \\
\text { (never or seldom) }\end{array}$ & 18235 & $56 \cdot 3^{*}$ & 5194 & $55 \cdot 3$ & 11211 & $57 \cdot 0$ & 1830 & $55 \cdot 7$ & 0.99 & $0.94,1.04$ & 0.98 & $0.91,1.06$ \\
\hline $\begin{array}{l}\text { Sets apart child's exercise time } \\
\text { (always, often or sometimes) }\end{array}$ & 26211 & $81 \cdot 1^{\star *}$ & 7536 & $80 \cdot 3$ & 16102 & $82 \cdot 0$ & 2573 & $78 \cdot 3$ & $0.82^{\star *}$ & $0.77,0.88$ & $0 \cdot 83^{\star *}$ & $0.75,0.91$ \\
\hline Restricts child's screen time (yes) & 29813 & $92 \cdot 3^{\star *}$ & 8753 & $93 \cdot 2$ & 18086 & $92 \cdot 1$ & 2974 & $90 \cdot 7$ & 0.96 & $0.87,1.06$ & $0.85^{\star}$ & $0.74,0.97$ \\
\hline \multicolumn{13}{|c|}{ Overweight children ( $n$ 11709) } \\
\hline $\begin{array}{l}\text { Prepares breakfast for child } \\
\text { (always, often or sometimes) }\end{array}$ & 10429 & $89 \cdot 7^{\star *}$ & 4566 & 91.5 & 5863 & $88 \cdot 3$ & \multicolumn{2}{|c|}{-} & $1 \cdot 34^{\star *}$ & $1 \cdot 17,1.54$ & & - \\
\hline $\begin{array}{l}\text { Stores soft drinks at home } \\
\text { (never or seldom) }\end{array}$ & 7972 & $68 \cdot 4$ & 3460 & $69 \cdot 3$ & 4512 & $67 \cdot 7$ & \multicolumn{2}{|c|}{-} & 1.01 & $0.93,1.10$ & & - \\
\hline $\begin{array}{l}\text { Buys soft drinks for child } \\
\text { (never or seldom) }\end{array}$ & 5287 & $45 \cdot 4^{*}$ & 2324 & $46 \cdot 5$ & 2963 & $44 \cdot 5$ & \multicolumn{2}{|c|}{-} & 1.02 & $0.94,1 \cdot 11$ & & - \\
\hline Exercises with child (>0h/d) & 6697 & $59 \cdot 9^{\star *}$ & 3002 & 63.0 & 3695 & $57 \cdot 6$ & \multicolumn{2}{|c|}{ - } & $1 \cdot 19^{* *}$ & $1.09,1.30$ & & - \\
\hline $\begin{array}{l}\text { Restricts child's exercise time } \\
\text { (never or seldom) }\end{array}$ & 6570 & $56 \cdot 4^{\star *}$ & 2738 & $54 \cdot 9$ & 3832 & $57 \cdot 6$ & \multicolumn{2}{|c|}{-} & 0.96 & $0.89,1.04$ & & - \\
\hline $\begin{array}{l}\text { Sets apart child's exercise time } \\
\text { (always, often or sometimes) }\end{array}$ & 9420 & $81 \cdot 1^{* *}$ & 4148 & $83 \cdot 2$ & 5272 & $79 \cdot 6$ & \multicolumn{2}{|c|}{-} & $1 \cdot 30^{\star *}$ & $1 \cdot 17,1.44$ & & - \\
\hline Restricts child's screen time (yes) & 10809 & 93.0 & 4654 & 93.4 & 6155 & $92 \cdot 7$ & \multicolumn{2}{|c|}{-} & 0.94 & $0.80,1 \cdot 11$ & & - \\
\hline
\end{tabular}

Differences in behaviour items between parental perception groups were evaluated using Pearson $\chi^{2}$ tests.

$\mathrm{OR}$ and $95 \% \mathrm{Cl}$ were derived from binary logistic regression analysis, in which the dependent variable is the single behaviour item. Both underestimation and overestimation groups are compared with the accurate estimation group, while adjusting for age, gender, region and school.

${ }^{\star} P<0.05,{ }^{\star \star} P<0.01$.

\section{Discussion}

The current national study indicated that nearly $40 \%$ of school-aged children and adolescents had their weight status misperceived by their parents, among which underestimation was much more prominent than overestimation. Higher probabilities of parental underestimation were found for younger children, boys and children with a lower BMI $Z$-score. The parents who perceived their child as having a healthy weight, whether accurately or not, tended to behave better on certain weight-related parenting behaviours. Moreover, the children who were perceived as having a healthy weight were more likely to behave healthily on certain weight-related behaviours.
Only $60 \cdot 8 \%$ of Chinese parents accurately perceived their child's weight, which was lower than the percentage in a representative sample of US children $(74 \%)^{(5)}$, indicating that Chinese parents should improve in identifying the weight of their child. Specifically, approximately $43 \%$ of the parents failed to recognize the overweight status of their child, although this value is lower than the estimated percentages from two meta-analyses (51 and 62\%, respectively $)^{(6,7)}$ and previous studies of Chinese children $(89 \text { and } 72 \% \text {, respectively })^{(16,17)}$. A traditional belief exists in China that being fat reflects affluence, which may have led Chinese mothers to consider that being overweight is not a big health issue ${ }^{(16)}$. Furthermore, the epidemic of overweight in Chinese children may have 'normalized' this health condition and contributed to parents' inability to 
Table 5 Associations between parental perception of their child's weight and the child's weight-related behaviours; children aged 6-17 years and their parents $(n$ 47 417) from seven provinces in China, September-November 2013

\begin{tabular}{|c|c|c|c|c|c|c|c|c|c|c|c|c|}
\hline \multirow[b]{3}{*}{ Behaviour item } & & & \multicolumn{6}{|c|}{ Parental perception } & & & & \\
\hline & \multicolumn{2}{|c|}{ Total } & \multicolumn{2}{|c|}{ Underestimate } & \multicolumn{2}{|c|}{ Accurate } & \multicolumn{2}{|c|}{ Overestimate } & \multicolumn{2}{|c|}{ Underestimate } & \multicolumn{2}{|c|}{ Overestimate } \\
\hline & $n$ & $\%$ & $n$ & $\%$ & $n$ & $\%$ & $n$ & $\%$ & OR & $95 \% \mathrm{Cl}$ & OR & $95 \% \mathrm{Cl}$ \\
\hline \multicolumn{13}{|l|}{ Underweight children (n 3144) } \\
\hline Fruits $(\geq 2$ servingst/d) & 724 & $24 \cdot 2^{*}$ & & - & 512 & $23 \cdot 1$ & 212 & $27 \cdot 6$ & & - & 1.05 & $0.84,1.32$ \\
\hline Vegetables ( $\geq 3$ servings $t / d$ ) & 590 & $19 \cdot 7$ & & - & 432 & $19 \cdot 4$ & 158 & $20 \cdot 7$ & & - & 1.05 & $0.82,1.34$ \\
\hline Meat products $(\leq 1$ serving $t / d)$ & 2094 & $70 \cdot 3^{\star \star}$ & & - & 1517 & 68.5 & 577 & 75.5 & & - & 1.03 & $0.82,1.30$ \\
\hline Breakfast (=7d/week) & 2572 & $84 \cdot 8^{\star *}$ & & - & 1947 & $86 \cdot 3$ & 625 & $80 \cdot 6$ & & - & 0.91 & $0.69,1.19$ \\
\hline Soft drinks ( $=0$ cup $/$ week) & 1067 & $36 \cdot 3$ & & - & 806 & $36 \cdot 7$ & 261 & $35 \cdot 1$ & & - & 1.04 & $0.84,1.29$ \\
\hline High-energy snacks ( $=0 \mathrm{~d} /$ week) & 740 & $24 \cdot 6$ & & - & 550 & 24.5 & 190 & 24.9 & & - & $1 \cdot 13$ & $0.90,1.43$ \\
\hline Fried food ( $=0$ time/week $)$ & 1351 & $45 \cdot 3^{* *}$ & & - & 1039 & $46 \cdot 8$ & 312 & $41 \cdot 1$ & & - & 0.94 & $0.77,1.15$ \\
\hline Western fast foods ( $=0$ time/week) & 1552 & $52 \cdot 1^{\star \star}$ & & - & 1122 & 50.5 & 430 & $56 \cdot 8$ & & - & 1.04 & $0.85,1.27$ \\
\hline MVPA $(\geq 1 \mathrm{~h} / \mathrm{d})$ & 816 & $29 \cdot 6$ & & - & 592 & $28 \cdot 7$ & 224 & $32 \cdot 4$ & & - & $1 \cdot 10$ & $0.87,1.38$ \\
\hline Screen time $(\leq 2 \mathrm{~h} / \mathrm{d})$ & 2102 & $78 \cdot 1^{\star \star}$ & & - & 1599 & $79 \cdot 3$ & 503 & 74.4 & & - & 0.89 & $0.68,1.17$ \\
\hline Homework time $(\leq 2 \mathrm{~h} / \mathrm{d})$ & 1963 & $66 \cdot 5^{\star *}$ & & - & 1429 & $65 \cdot 0$ & 534 & $70 \cdot 8$ & & - & $1 \cdot 10$ & $0.86,1.39$ \\
\hline \multicolumn{13}{|l|}{ Healthy-weight children ( $n$ 32 564) } \\
\hline Fruit ( $\geq 2$ servings/d) & 7820 & $25 \cdot 2^{* *}$ & 2102 & 23.4 & 4922 & $25 \cdot 9$ & 796 & $25 \cdot 9$ & $0.89^{\star *}$ & $0.84,0.95$ & 0.91 & $0.83,1.00$ \\
\hline Vegetable ( $\geq 3$ servings/d) & 5984 & $19 \cdot 3^{\star \star}$ & 1641 & $18 \cdot 2$ & 3678 & 19.4 & 665 & 21.5 & 0.97 & $0.90,1.03$ & 1.01 & $0.92,1.12$ \\
\hline Meat products ( $\leq 1$ servings $/ d$ ) & 22676 & $73 \cdot 2^{\star \star}$ & 6421 & 71.5 & 13914 & 73.5 & 2341 & $76 \cdot 3$ & $0.94^{*}$ & $0.88,1.00$ & 0.98 & $0.89,1.08$ \\
\hline Breakfast (=7d/week) & 26552 & $84 \cdot 6^{\star *}$ & 7859 & $86 \cdot 3$ & 16259 & 84.7 & 2434 & 78.4 & 0.96 & $0.89,1.04$ & $0 \cdot 83^{\star *}$ & $0.75,0.92$ \\
\hline Soft drinks ( $=0$ cup $/$ week $)$ & 10385 & 34.1 & 2992 & 33.9 & 6424 & 34.4 & 969 & 32.5 & 0.95 & $0.90,1.00$ & 0.93 & $0.85,1.01$ \\
\hline High-energy snacks ( $=0 \mathrm{~d} /$ week) & 7785 & $25 \cdot 0^{\star}$ & 2316 & $25 \cdot 6$ & 4754 & 24.9 & 715 & $23 \cdot 3$ & 1.00 & $0.94,1.06$ & 0.93 & $0.84,1.02$ \\
\hline Fried food ( $=0$ time/week) & 13391 & $43 \cdot 1^{\star \star}$ & 3967 & $44 \cdot 0$ & 8261 & 43.5 & 1163 & $37 \cdot 8$ & 0.96 & $0.91,1.01$ & $0 \cdot 89^{\star \star}$ & $0.82,0.97$ \\
\hline Western fast foods ( $=0$ time/week) & 16041 & $51 \cdot 7^{\star \star}$ & 4564 & $50 \cdot 7$ & 9799 & $51 \cdot 7$ & 1678 & 54.8 & 0.96 & $0.91,1.01$ & 0.95 & $0.87,1.03$ \\
\hline MVPA $(\geq 1 \mathrm{~h} / \mathrm{d})$ & 8809 & $30 \cdot 8^{*}$ & 2441 & 29.5 & 5503 & 31.4 & 865 & $31 \cdot 0$ & $0.89^{\star \star}$ & $0.84,0.95$ & 0.96 & $0.88,1.06$ \\
\hline Screen time $(\leq 2 \mathrm{~h} / \mathrm{d})$ & 21471 & $77 \cdot 1^{* *}$ & 6167 & $77 \cdot 0$ & 13382 & 77.9 & 1922 & $72 \cdot 2$ & 0.95 & $0.89,1.02$ & $0.85^{\star *}$ & $0.77,0.94$ \\
\hline Homework time $(\leq 2 \mathrm{~h} / \mathrm{d})$ & 20321 & $66 \cdot 6^{\star *}$ & 6151 & $69 \cdot 8$ & 12302 & $65 \cdot 8$ & 1868 & 61.9 & 1.00 & $0.94,1.06$ & $0.87^{\star *}$ & $0.79,0.96$ \\
\hline \multicolumn{13}{|l|}{ Overweight children ( $n$ 11 709) } \\
\hline Fruits ( $\geq 2$ servings/d) & 3022 & $27 \cdot 1^{*}$ & 1348 & $28 \cdot 3$ & 1674 & $26 \cdot 2$ & & - & 1.06 & $0.97,1.17$ & & - \\
\hline Vegetables ( $\geq 3$ servings/d) & 2297 & $20 \cdot 6$ & 981 & $20 \cdot 6$ & 1316 & $20 \cdot 5$ & & - & 0.97 & $0.88,1.08$ & & - \\
\hline Meat products ( $\leq 1$ servings $/ d$ ) & 8171 & $73 \cdot 5^{\star \star}$ & 3596 & $76 \cdot 0$ & 4575 & 71.6 & & - & $1 \cdot 17^{\star \star}$ & $1.06,1.29$ & & - \\
\hline Breakfast (=7d/week) & 9633 & 85.5 & 4128 & $86 \cdot 1$ & 5505 & $85 \cdot 0$ & & - & 1.06 & $0.94,1.20$ & & - \\
\hline Soft drinks ( $=0$ cup/week) & 3588 & $32 \cdot 8^{\star \star}$ & 1648 & $35 \cdot 4$ & 1940 & $30 \cdot 8$ & & - & $1 \cdot 11^{*}$ & $1.01,1.21$ & & - \\
\hline High-energy snacks ( $=0 \mathrm{~d} /$ week) & 3052 & $27 \cdot 3$ & 1282 & $27 \cdot 0$ & 1770 & 27.5 & & - & 0.95 & $0.86,1.04$ & & - \\
\hline Fried food ( $=0$ time/week) & 4739 & $42 \cdot 4$ & 1983 & $41 \cdot 7$ & 2756 & 42.9 & & - & 0.97 & $0.89,1.06$ & & - \\
\hline Western fast foods ( $=0$ time/week) & 5823 & $52 \cdot 2$ & 2448 & $51 \cdot 7$ & 3375 & $52 \cdot 6$ & & - & $0.91^{*}$ & $0.84,0.99$ & & - \\
\hline MVPA $(\geq 1 \mathrm{~h} / \mathrm{d})$ & 3390 & $32 \cdot 8^{\star}$ & 1379 & 31.5 & 2011 & 33.8 & & - & 1.00 & $0.91,1.10$ & & - \\
\hline Screen time $(\leq 2 \mathrm{~h} / \mathrm{d})$ & 7910 & $77 \cdot 3^{\star \star}$ & 3425 & $78 \cdot 6$ & 4485 & $76 \cdot 4$ & & - & $1 \cdot 18^{\star *}$ & $1.06,1.31$ & & - \\
\hline Homework time $(\leq 2 \mathrm{~h} / \mathrm{d})$ & 7433 & $67 \cdot 4^{\star \star}$ & 3311 & 70.5 & 4122 & $65 \cdot 1$ & & - & 1.06 & $0.96,1.16$ & & - \\
\hline
\end{tabular}

MVPA, moderate-to-vigorous physical activity.

Differences in behaviour items between parental perception groups were evaluated using Pearson $x^{2}$ tests.

$\mathrm{OR}$ and $95 \% \mathrm{Cl}$ were derived from binary logistic regression analysis, in which the dependent variable is the single behaviour item. Both underestimation and overestimation groups are compared with the accurate estimation group, adjusting for age, gender, region and school.

${ }^{*} P<0.05,{ }^{*} P<0.01$.

†A serving of fruit or vegetable is equivalent to $100 \mathrm{~g}$, whereas a serving of meat product is equivalent to $75 \mathrm{~g}$.

recognize that their child is overweight ${ }^{(16)}$. Parental unawareness of their child's overweight status draws attention since it may pose barriers for parents to take measures to address the problem. Moreover, parental underestimation may influence overweight children's self-assessment of their weight, as well as their desire to lose weight and improve their lifestyle ${ }^{(26)}$. In addition, a substantial proportion $(29 \%)$ of parents underestimated the healthyweight status of their child, which is much higher than the percentages in a meta-analysis $(14 \%)^{(7)}$ and a US study $(10 \%)^{(5)}$. Parental underestimation of healthy-weight children also warrants attention, because this may lead parents to encourage their child to consume extra food to facilitate weight gain, thereby elevating the child's risk of future obesity ${ }^{(27)}$. We observed a similar distribution in weight perception between the children themselves and their parents (approximately $68 \%$ of children received concordant perceptions), indicating that weight perception from parents and children were closely related, which was supported by previous studies ${ }^{(18,26)}$. Interventions targeting either parental or self-perception of child weight should take the potential interaction into account.

When assessing the correlates of parental underestimation, we found that parents were more likely to underestimate the weight status of younger children, which was supported by other studies ${ }^{(6-8)}$. A possible explanation is that weight increase in younger children indicates good nutrition and parents believe that their child could grow out of the excess weight condition. In a qualitative study, low-income mothers described overweight pre-school children as 'thick' or 'solid' and tended to not believe that the children were overweight so long as 
they were physically active and had a good appetite ${ }^{(28)}$. Another study also demonstrated that parents tended to normalize their pre-school child's excess body weight as 'toddler pudge' or 'cute baby fat ${ }^{\text {(29) }}$. Considering that overweight in early childhood can persist into later life ${ }^{(30)}$, early identification of overweight may present crucial opportunities for obesity prevention and intervention.

Furthermore, parents were more likely to underestimate the weight of boys than of girls, which was consistent with some ${ }^{(7,31)}$ but not all literature ${ }^{(12)}$. Although this trend may be affected by gender difference in body composition ${ }^{(32)}$, it is more possible to be explained by differential social norms about the ideal body size for boys and girls. Healthy-weight girls tend to be perceived as having a healthy weight or even slightly overweight according to the social desire for slimness, whereas healthy-weight boys may be considered thin because the expectation for boys is that they should be big and strong ${ }^{(7)}$. Boys perceived as underweight may be encouraged to eat a substantial amount of food to gain a larger body size, placing them at a higher risk of being overweight.

Parents tend to be most influential in shaping the lifestyle of their child, especially their eating behaviours and determining what food is available for them. In our study, it was noticed that over half of the parents bought soft drinks for their child and that nearly one-third stored soft drinks at home. Given that the consumption of sugarsweetened beverages has been proved to be positively associated with obesity ${ }^{(33)}$, Chinese parents should be educated about the harmfulness of soft drinks.

Although parents' awareness of their child's weight is suggested to be an important first step for the child's enrolment in an obesity management programme ${ }^{(3)}$, the relationship between parental perception of child weight and weight-related parenting behaviours was seldom discussed $^{(12,13,18)}$. Contrary to popular belief, we found that parents who accurately recognized that their child was overweight were less likely to prepare breakfast for them, exercise with them and set apart exercise time for them. Similarly, parents who misperceived their healthy-weight child as being overweight were less likely to prepare breakfast for them, exercise with them, set apart exercise time for them and restrict their screen time, but were more likely to store soft drinks for them. These results imply that parental recognition that their child is overweight, accurate or not, may be an insufficient motivation to change parenting behaviours regarding their child's weight. Alternatively, those who accurately perceived their child's overweight status may have improved their weight-related parenting behaviours, but the changes were too slight to be detected. Jaballas et al. reported that $40 \%$ of parents who considered their child as overweight believed that overweight was a condition that would be outgrown ${ }^{(34)}$. Psychological factors such as belief and confidence may also mediate in the association between the perception and implementation of behaviours. Parents who perceive their child as overweight may lack intention to improve their child's weight or do not believe that they can change it through effort. Another possible explanation is that an accurate identification of their child being overweight is not a prerequisite for parents to improve weight-related health behaviours, which may likely be universal to all parents (i.e. most parents want their child to be healthy $)^{(12)}$. Thus, more research is warranted to clarify the associations between parental perception and weight-related parenting behaviours and to explore the potential barriers.

Regarding the child's weight-related behaviours, overweight children who were accurately recognized by parents tended to consume more meat products, soft drinks and spend more screen time than those who were underestimated by parents. In addition, children of healthy weight but wrongly considered overweight were more likely to skip breakfast, consume fried food, and spend more screen and homework time. A hypothesis is that children who are perceived by parents as overweight may have certain undesirable behavioural characteristics themselves, such as consuming more meat and fried food. A qualitative study by Jain et al. revealed that children were not believed to be overweight by their mother if they were active and had a healthy diet ${ }^{(28)}$. Furthermore, two longitudinal studies in Dutch and Australian children showed that parental identification of their child being overweight was not protective against further weight gain $^{(35,36)}$. An important gap exists between parental recognition of their child's overweight/obesity and improvement in weight-related parenting behaviours or child behaviours. This implies that health education targeting parents to correctly identify their child's overweight or obesity alone is not sufficient to improve parenting behaviours and child behaviours. Therefore, future obesity intervention involving parents should motivate them on how to promote healthy behaviours of their children or directly engage children in healthy diet and exercise with the involvement of their parents. Although parents hold responsibility of lifestyle guidance for their child, the wider environment beyond the family can still weaken parental influences. Digital marketing techniques using social networking software and mobile phone apps give food and beverage industries access to children without parental oversight ${ }^{(37)}$.

From another point of view, parental underestimation of healthy-weight children was negatively associated with the children consuming more fruits and less meat products and taking more MVPA. This should be taken into consideration, since less fruit and more meat intake and shorter time of MVPA might lead to higher obesity risk. Yet, given the current cross-sectional design, additional research using longitudinal data is needed to provide better insight into the influence of parental perception of their child's weight on the child's weight-related behaviours over time. 
The current study presents two strengths. First, we investigated parental perception of child's weight in a large national sample in Chinese children. Second, we examined parental perception of child's weight in relation to both weight-related parenting behaviours and child's behaviours, which has been investigated only in very limited studies. Since a considerable proportion of parents misperceived their child's actual weight, it makes sense to examine the relationship between parental misperception and parenting behaviours and child's behaviours that could impact child's weight. Nevertheless, limitations should be considered when interpreting the findings. First, the cross-sectional design determines that one should be very careful when examining the directionality of associations between parental misperception and weightrelated parenting behaviours or child behaviours. Second, children's weight-related behaviours were measured with questionnaires, which might lead to information bias.

\section{Conclusion}

Parental underestimation of child's weight was prominent among healthy-weight and overweight Chinese children. Higher probabilities of parental underestimation were found among younger children, boys and children with a lower BMI Z-score. Parents' correct recognition that their child was overweight did not appear to translate into healthier changes in weight-related parenting behaviours or the child's behaviours. Future obesity intervention involving parents should motivate them on how to promote their child's healthy behaviours or directly engage the child in healthy diet and exercise with parents' involvement.

\section{Acknowledgements}

Acknowledgements: The authors acknowledge the Guangzhou Health Care Clinics of Middle and Primary Schools for their contribution in the data collection. They also thank the participants in the survey and the postgraduates for conducting the questionnaire survey. Financial support: This work was supported by Guangdong Science and Technology Program (No. 2016A020225002), Guangdong Natural Science Fund (No. 2015A030313093) and the Research Special Fund for Public Welfare of Health from the Ministry of Health, People's Republic of China (No. 201202010). The funders had no role in the design, analysis or writing of this article. Conflict of interest: The authors declare that they have no competing interests. Authorship: J.J. and J.M. supervised the study. T.Z. and L.M. performed the data collection. T.Z. analysed the data and drafted the manuscript. L.C. guided the analysis and interpretation of data and critically revised the manuscript. Y.C. designed the study, provided suggestions in the analysis and approved the final manuscript. All authors read and approved the submission of the final manuscript. Ethics of human subject participation: This study was conducted according to the guidelines laid down in the Declaration of Helsinki and all procedures involving human subjects were approved by the Ethics Committee of Peking University. Written informed consent was obtained from all subjects.

\section{Supplementary material}

To view supplementary material for this article, please visit https://doi.org/10.1017/S136898001800006X

\section{References}

1. Lobstein T, Jackson-Leach R, Moodie ML et al. (2015) Child and adolescent obesity: part of a bigger picture. Lancet $\mathbf{3 8 5}$, 2510-2520.

2. Johannsen DL, Johannsen NM \& Specker BL (2006) Influence of parents' eating behaviors and child feeding practices on children's weight status. Obesity (Silver Spring) 14, 431-439.

3. Davidson K \& Vidgen H (2017) Why do parents enrol in a childhood obesity management program?: a qualitative study with parents of overweight and obese children. BMC Public Health 17, 159.

4. Regber S, Novak M, Eiben G et al. (2013) Parental perceptions of and concerns about child's body weight in eight European countries - the IDEFICS study. Pediatr Obes 8, 118-129.

5. Chen HY, Lemon SC, Pagoto SL et al. (2014) Personal and parental weight misperception and self-reported attempted weight loss in US children and adolescents, National Health and Nutrition Examination Survey, 2007-2008 and 2009-2010. Prev Chronic Dis 11, E132.

6. Rietmeijer-Mentink M, Paulis WD, van Middelkoop M et al. (2013) Difference between parental perception and actual weight status of children: a systematic review. Matern Child Nutr 9, 3-22.

7. Lundahl A, Kidwell KM \& Nelson TD (2014) Parental underestimates of child weight: a meta-analysis. Pediatrics 133, e689-e703.

8. Hudson E, McGloin A \& McConnon A (2012) Parental weight (mis)perceptions: factors influencing parents' ability to correctly categorise their child's weight status. Matern Child Health J 16, 1801-1809.

9. Manios Y, Moschonis G, Karatzi K et al. (2015) Large proportions of overweight and obese children, as well as their parents, underestimate children's weight status across Europe. The ENERGY (EuropeaN Energy balance Research to prevent excessive weight Gain among Youth) project. Public Health Nutr 18, 2183-2190.

10. Aljunaibi A, Abdulle A \& Nagelkerke N (2013) Parental weight perceptions: a cause for concern in the prevention and management of childhood obesity in the United Arab Emirates. PLoS One 8, e59923.

11. de Hoog ML, Stronks K, van Eijsden M et al. (2012) Ethnic differences in maternal underestimation of offspring's weight: the ABCD study. Int J Obes (Lond) 36, 53-60.

12. Remmers T, van Grieken A, Renders CM et al. (2014) Correlates of parental misperception of their child's weight status: the 'Be Active, Eat Right' study. PLoS One 9, e88931.

13. Al-Qaoud NM, Al-Shami E \& Prakash P (2010) Kuwaiti mothers' perception of their preschool children's weight status. J Dev Behav Pediatr 31, 505-510. 
14. Almoosawi S, Jones AR, Parkinson KN et al. (2016) Parental perception of weight status: influence on children's diet in the Gateshead millennium study. PLoS One 11, e0144931.

15. Magarey AM, Perry RA, Baur LA et al. (2011) A parent-led family-focused treatment program for overweight children aged 5 to 9 years: the PEACH RCT. Pediatrics 127, 214-222.

16. Chen S, Binns CW, Maycock B et al. (2014) Chinese mothers' perceptions of their child's weight and obesity status. Asia Pac J Clin Nutr 23, 452-458.

17. Yao NL \& Hillemeier MM (2012) Weight status in Chinese children: maternal perceptions and child self-assessments. World J Pediatr 8, 129-135.

18. Wen X \& Hui SS (2011) Chinese parents' perceptions of their children's weights and their relationship to parenting behaviours. Child Care Health Dev 37, 343-351.

19. Institute of Child and Adolescent Health of Peking University (2012) The Health Information Platform of Primary and Secondary School Students of Plan 1147. http:// www.health1147.bjmu.edu.cn/ (accessed January 2017).

20. Chen Y, Ma L, Ma Y et al. (2015) A national school-based health lifestyles interventions among Chinese children and adolescents against obesity: rationale, design and methodology of a randomized controlled trial in China. BMC Public Health 15, 210.

21. Chen C \& Lu FC, Department of Disease Control, Ministry of Health, People's Republic of China (2004) The guidelines for prevention and control of overweight and obesity in Chinese adults. Biomed Environ Sci 17, Suppl., 1-36.

22. National Health and Family Planning Commission of the People's Republic of China (2014) Screening Standard for Malnutrition of School-age Children and Adolescents. http:// www.moh.gov.cn/zwgkzt/pqt/201407/38b15c0a1ed444e89 08e12752decaffa.shtml (accessed January 2017).

23. Group of China Obesity Task Force (2004) Body mass index reference norm for screening overweight and obesity in Chinese children and adolescents. Zhonghua Liu Xing Bing Xue Za Zbi 25, 97-102.

24. World Health Organization (2007) Growth Reference 5-19 Years. Geneva: WHO; available at http://www.who.int/ growthref/who2007_bmi_for_age/en/

25. de Onis M, Onyango AW, Borghi E et al. (2007) Development of a WHO growth reference for school-aged children and adolescents. Bull World Health Organ 85 , 660-667.

26. Huang JS, Donohue M, Becerra K et al. (2009) Relationship between parents' and children's weight perceptions results of a survey. Infant Child Adolesc Nutr 1, 15-20.

27. Tschamler JM, Conn KM, Cook SR et al. (2010) Underestimation of children's weight status: views of parents in an urban community. Clin Pediatr (Phila) 49, 470-476.

28. Jain A, Sherman SN, Chamberlin LA et al. (2001) Why don't low-income mothers worry about their preschoolers being overweight? Pediatrics 107, 1138-1146.

29. Eli K, Howell K, Fisher PA et al. (2014) 'A little on the heavy side': a qualitative analysis of parents' and grandparents' perceptions of preschoolers' body weights. BMJ Open 4, e006609.

30. Singh AS, Mulder C, Twisk JWR et al. (2008) Tracking of childhood overweight into adulthood: a systematic review of the literature. Obes Rev 9, 474-488.

31. Li J, Lei J, Wen S et al. (2014) Sex disparity and perception of obesity/overweight by parents and grandparents. Paediatr Child Health 19, e113-e116.

32. Karlsson AK, Kullberg J, Stokland E et al. (2013) Measurements of total and regional body composition in preschool children: a comparison of MRI, DXA, and anthropometric data. Obesity (Silver Spring) 21, 1018-1024.

33. Malik VS, Popkin BM, Bray GA et al. (2010) Sugar-sweetened beverages, obesity, type 2 diabetes mellitus, and cardiovascular disease risk. Circulation 121, 1356-1364.

34. Jaballas E, Clark-Ott D, Clasen C et al. (2011) Parents' perceptions of their children's weight, eating habits, and physical activities at home and at school. J Pediatr Health Care 25, 294-301.

35. Gerards SM, Gubbels JS, Dagnelie PC et al. (2014) Parental perception of child's weight status and subsequent BMIz change: the KOALA birth cohort study. BMC Public Health 14, 291.

36. Robinson E \& Sutin AR (2016) Parental perception of weight status and weight gain across childhood. Pediatrics 137, e20153957.

37. World Health Organization (2013) Marketing of Foods High in Fat, Salt and Sugar to Children: Update 2012-2013. Copenhagen: WHO Regional Office for Europe. 